\title{
ESTRATEGIAS Y PERSPECTIVAS DOCUMENTALES EN LA INFORMACIÓN DIGITAL
}

\author{
Juan C. Marcos \\ jmarcos@ccinf.ucm.es \\ Concha Edo \\ conchaed@ccinf.ucm.es \\ Jesús Flores \\ jmflores@cinf.ucm.es \\ Pedro García-Alonso \\ pedrog_a@yahoo.com \\ David Parra \\ dparra@influenceandprofit.com
}

(Universidad Complutense de Madrid)

\section{Resumen:}

La información digital es ya una realidad y la creación de este tipo de información es cada vez mayor, por lo que se hace necesario un control, un análisis y una gestión; y en este trabajo se proponen fórmulas para entender la información digital y sus características. La documentación hace posible el seguimiento y el estudio del proceso documental aplicado a las informaciones que facilitan los medios de comunicación, que aportan los elementos informativos necesarios para crear fondos documentales con sus noticias. Por su parte, el lector, se constituye en elemento creativo de la información, aportando su experiencia personal.

Palabras clave: Periodismo digital, documentación, medios de comunicación, lector, documentalista.

\section{Abstract:}

Digital information is already a reality and the creation of this type of information is continuously growing and so it has become necessary to establish certain controls, analyses and organizational procedures. Formulas are proposed in this text for understanding digital information and its main characteristics. The documentary process is studied as applied to the information furnished by the communications media. In this process, the communications media furnish the necessary informative elements in order to create documentary archives and collections with their news. In turn, the reader becomes a creative element in the information by furnishing his or her personal experience.

Key words: Digital journalism, documentation, media, readers, researcher.

\footnotetext{
1 Este texto forma parte de los trabajos correspondientes al proyecto "Incidencia de Internet en los medios de comunicación españoles: Informe anual 2007", financiado por la Comunidad de Madrid y la Universidad Complutense.
} 


\section{MÁS POR MENOS: EL RETO DE LA BUENA INFORMACIÓN}

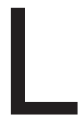

a documentación es, sin duda, la información elevada al cuadrado, o lo que es lo mismo, la información que ofrece nueva información. No se trata de un juego de palabras. Es algo más, un valor añadido que se suma a la aportación del periodista. Durante siglos, la carencia de contenidos era el reflejo de una sociedad en la que no abundaban los materiales y en la que los índices de lectura eran muy escasos. Pasado ese paréntesis, los días presentes determinan todo lo contrario: abundan los soportes (prensa escrita y digital, radio, televisión, cine, publicidad, teléfonos móviles y, como no, Internet) y el aumento de los lectores con el crecimiento de la población y las facilidades para acceder a los contenidos informativos. Y lo que es peor, o mejor dirán otros, la superabundancia de la información que imposibilita su acceso, seguimiento y control si no se cuenta con un especialista que la analice, la estudie, la clasifique y la ponga al servicio del consumidor, bien un lector, un redactor, un investigador o un joven estudiante.

No es nada nuevo. La referencia principal es el documentalista. Quizás el nombre es más reciente, pero la figura que lleva a cabo esas tareas ha existido siempre. La información es un acto social que contribuye a mejorar la vida de las personas. Así, se entiende la necesidad de conservarla. Aparecen en un primer momento las bibliotecas, como lugar de acopio de materiales y del saber, que siguen en la actualidad cumpliendo parte de esas funciones. Con la llegada del siglo XX, la documentación tomó el relevo para agilizar el proceso de análisis de los documentos, de tal forma que estuvieran listos cada vez que un usuario pedía la información.

Cuando los demandantes de información son los periodistas, la situación no cambia significativamente. Hace ya más de un siglo que Pulitzer creo el Servicio de Referencia del The New York Times evaluando las perspectivas de futuro que la propia información generaba sobre sí misma. El objetivo era hacer índices para facilitar la tarea de recuperar los contenidos y que los propios periodistas utilizaran sus informaciones para crear nuevos elementos informativos. Quedó establecida entonces una máxima documental en el periódico que luego será considerada la Biblia de los medios escritos: la información analizada y estructurada documentalmente sirve para generar nuevas informaciones. Desde entonces, la mayoría de los periodistas lo saben y lo aplican. Pero no siempre ha sido así.

El fin último de los medios de comunicación es transmitir una información clara, objetiva, veraz y en el menor tiempo posible a sus usuarios. Esta fórmula fue exitosa durante casi todo el siglo XX, pero la llegada de las tecnologías dio un giro a la forma y al fondo de la información. Los modos y maneras dieron al traste con más de dos siglos de hacer periodismo, algo que no sentó bien en algunas redacciones, acostumbradas a sistemas de edición e impresión totalmente controlados por el hombre. La presencia cada vez mayor de los ordenadores en las redacciones fue el detonante para los cambios que se avecinaban. Al final, la implantación sin vuelta atrás 
de Internet, fue la excusa perfecta para dar el salto definitivo hacia un público que pedía una nueva fórmula, un diseño diferente y un mejor contenido de la información.

Todo en esta (r)evolución ha ido adaptándose a la nueva realidad. Transcurrida ya más de una década de los periódicos digitales españoles en Internet, pasado el primer escollo de comparar lo impreso con lo digital, metidos de lleno en el fenómeno de los blogs con todas sus variantes, el siguiente paso está dándose hacia una comunicación total en la que la documentación tiene y debe aportar sus recursos para mejorar los contenidos informativos y garantizar su credibilidad. Sin documentación no hay buena información. Partiendo de esa premisa, se ha de evaluar qué es y hacia dónde se encamina la información y de qué forma la documentación estará al lado de los periodistas creativos, es decir aquellos que plantean la información como un elemento global en el que caben datos, ideas, opiniones, estudios, resultados y evaluaciones. Ese ofrecimiento, colocado en Internet de forma rápida y con un buen diseño, debería bastar para crear una serie de lectores fieles, que eligen esa opción frente a miles de posibles alternativas informativas. Se trata, en definitiva, de dar más información por menos (tiempo-espacio), ya que el lector tiene menos tiempo y más opciones para leer otros periódicos digitales o acudir a medios audiovisuales que sirven la información al momento.

\section{LAS CARACTERÍSTICAS DE LA INFORMACIÓN EN LÍNEA}

Como punto de partida conviene recordar una expresión bíblica: "Ay de aquel..." editor que siga creyendo que la información lo es todo. Y lo mismo pasa con la documentación. Más allá, también, hay vida, sobre todo cuando el lector decide. Aquellos tiempos quedaron atrás, como el siglo XX. Ahora, la información es -o debería ser- dinámica, ágil, arriesgada, entretenida y con muchos e interesantes datos o puntos de vista, fruto de un trabajo de investigación y documentación, del empleo de diversas fuentes $y$, por supuesto, de los contactos de cada periodista. La verticalidad de la información dio paso a la horizontalidad en la que el lector recurre a muchos enlaces puestos a su disposición para completarla o simplemente para criticarla. Es una simbiosis en la que cohabitan el periodista y el lector en un mismo espacio físicovirtual.

Antes de que en España fueran apareciendo los periódicos digitales, ya la documentación había establecido algunas bases teóricas para su funcionamiento. Entonces se formuló la figura del Nuevo Comunicador (Marcos Recio, 1999: 80-84) que ha terminado por establecerse como el periodista que aglutina diversas tareas, apoyadas todas ellas en los recursos que la tecnología pone a su disposición. En 1999, ese comunicador era el Periodista de Información Electrónica (PIE), al que le suministraba datos y le ayudaba en su recolección el Documentalista de Información Electrónica (DIE). Ambos formaban el lado principal de un triángulo que completaba el experto en la información, aportando puntos de vista diferentes, siempre complementarios a la tarea creativa del periodista y el lector, pero éste último se analiza en el epígrafe 4. 
Era y es un nuevo reto que afronta la documentación, impulsada por un factor creciente de cantidad frente a calidad, que el lector aprecia cuando la información se le suministra estructurada y con contenidos interesantes. Tener información ya no es suficiente. Lo importante es que el periodista cuente con los recursos suficientes para mejorar dichos contenidos y los encuentre preparados y de manera rápida:

La acumulación constante de información y la cada vez más numerosa publicación de informes, trabajos de investigación e incluso simples publicaciones con ideas importantes para tener conocimiento de cualquier campo, obligó a las empresas de prensa a crear centros propios que trabajaran con todo ese material, con una única idea: prepararlo cuando lo solicitara el redactor (Marcos Recio, 1999: 8081).

¿Cómo se ha llegado a esta situación? La comunicación siempre ha fluido en una única dirección: periodista que crea contenidos para el lector. En ese proceso, el documentalista tiene parte de responsabilidad, ofreciendo datos para mejorar el producto informativo. Sin embargo, el desarrollo de los medios y sobre todo la evolución de la tecnología rompió esa línea y con ella la de la escasa participación de los lectores (cartas al director y algún que otro artículo) para colocar en el pedestal al dios supremo de la información: el lector.

Los periodistas son conscientes de que ésa era su meta. Dar servicio al lector, ofrecerle información útil, datos de interés e incluso teorías sobre aspectos apenas conocidos cuando se acercaba a la información. Pero la realidad no siempre ha sido así. Hasta épocas muy recientes, donde la competencia informativa era escasa, el lector importaba menos. Ahora, cuando puede elegir, los editores saben que sin un buen producto informativo no se consiguen lectores. ¿Qué es un buen producto informativo? Es fácil de plantear y complicado de resolver. Una propuesta inicial sería información más servicios, puesto que la mayoría de medios, así lo hacen. Tiene que haber más y no nos referimos ahora a los utensilios de todo tipo que se regalan con los periódicos. Una información de calidad es aquella que ofrece todas las perspectivas posibles para el lector y además le abre otras puertas para que él decida cómo ha de enfrentarse a la hora de leerla. Atrás quedó la manera de contar las historias, en la que se daban muchos datos y cuanto antes mejor. Hoy la información es un cúmulo de acciones propias del que la redacta, del que la planifica (entiéndase aquí como el sitio donde se coloca) y del que la recibe, que a su vez la alimenta con sus propios comentarios y con un nuevo enfoque que el periodista reutiliza. Es una especie de cordón umbilical en el que el periodista recupera parte de lo entregado para avanzar hacia otros contenidos o para mejorar los presentes. Hay una comunicación importante entre ambos y eso le permite al periodista saber si va por el buen camino.

Por otra parte, existe ya una segmentación de perfiles de usuario dentro del universo periodístico que posibilita al redactor la creación de contenidos que sí le interesan al lector y a otros posibles que lleguen a través de enlaces. Ya hace tiempo que se viene hablando de la personalización de contenidos, -información a la medida de 
los lectores- como la mejor oferta para conseguir nuevos lectores en los medios digitales y para mantener a los que ya son lectores habituales.

Pero, la información en línea es mutante como la propia tecnología que la sustenta. Es cierto que las necesidades de cada usuario en Internet son diferentes, pero se han conseguido perfiles adaptados a colectivos interesados en una determinada información. En todo caso, siempre difíciles de mantener, sobre todo si los contenidos no muestran diversos frentes informativos, ágiles y actualizados. Así, los periódicos digitales tuvieron apenas una década para descubrir e implantar un tipo de información desconocido hasta entonces. Y, cuando creen que han sentado las bases de una nueva filosofía periodística, se dan cuenta de que hay otras realidades a la vuelta de cada nueva edición. Quizás, algunos ejemplos aclaren esta situación: el año pasado, apareció la segunda generación de Internet (Web 2.0, también llamada Internet Social) que se caracterizó por un triunfo de algunos sitios de comunicación de tipo social, por la implicación de un número considerable de personas que acuden a esos sitios web, como por ejemplo YouTube, MySpace, LinkedIn o el fenómeno de los blogs. Conviene recordar que la información también es tiempo de consumo frente o con una pantalla. Así, mientras los lectores descargan vídeos gratis de YouTube no leen informaciones y mientras apuestan por crear su propio blog, tampoco acceden a contenidos informativos. Pero queda la esperanza. Que las nuevas generaciones encuentren un sentido a la información total, es decir, la que les ofrece elementos suficientes para mantenerse perfectamente informados. De esta forma, 2007 será el de la Web 3.0 o Web Semántica, que busca de acuerdo con el creador de la WWW, Tim Berners-Lee, añadir significado a la web, de manera que, en lugar de un mero catálogo, se convierta en una guía 'inteligente', con sitios capaces de intercambiar información sobre sus contenidos (Berners-Lee; Hendler; Lassila, 2001). Por tanto, no solo bastará con poner la información, sino que habrá que crear un lugar donde los lectores se sientan a gusto porque además hay servicios, opiniones, espacios críticos, comunidades de usuario, puntos de encuentro, debates, entretenimiento, juegos y todo aquello que complementa una buena información para hacer que el lector se sienta que forma parte de ese medio porque él es la razón de ser de la información.

\section{LOS RECURSOS DOCUMENTALES APLICADOS A LA INFORMACIÓN DIGI- TAL}

Mientras la información se adapta a la nueva realidad que supone lo digital, la documentación hace ya tiempo que viene estableciendo un orden de prioridades en su funcionamiento. Los centros de documentación tardaron en acceder a la tecnología, pero, una vez dentro, supieron adaptarse a las necesidades que los redactores les demandaban. En medio, un desierto para adaptar el proceso documental al ritmo de trabajo de los medios de comunicación.

Algunas informaciones están planificadas; es decir, se sabe con anterioridad cuándo se van a celebrar. Sin embargo, otras son las que dan origen a la noticia y 
nadie sabe cuándo se van a convertir en eso. En ambos casos, en el día a día, en lo que va sucediendo en cada momento y en lo que es previsible, el documentalista trabaja con el fin de poner a disposición de los informantes las fuentes necesarias para completar el proceso informativo. Sin un seguimiento, una pista, un análisis, una implicación y una metodología, el resultado final de la información no es completo. En este sentido, Nathan consiguió ya hace tiempo valorar la unión que existe entre la documentación y la propia información que se genera antes y después de crearla y precisaba bien el origen de la información cuando afirmaba que el periódico mantiene sus dedos en el pasado y sus ojos en el futuro. En la noticia "muerta" quedan pistas tan importantes del futuro como explicaciones del pasado, y es un documento indeleble, infalible, que sirve de guía para que las opiniones del experto director estén bien encaminadas (Nathan, 1910: 597-599).

Tradicionalmente, el sistema establecido funcionaba y era dinámico. Los centros de documentación servían como apoyo a la labor periodística. Su funcionamiento requería una consulta por parte del redactor a la que da servicio el documentalista. La llegada de la tecnología favoreció el trabajo creando intranets en los medios que permitían a los periodistas hacer sus propias búsquedas, tanto en su centro como en consultas externas a bases de datos, generalmente de pago. El sistema no era perfecto, pero funcionaba. Y lo pueden atestiguar miles de periodistas que han venido trabajando de esta forma.

Se produce un giro radical cuando a los medios impresos les sale competencia digital, tanto de su hermano mayor como de otros periódicos en Internet. Así, en esta red se engloba la nueva fórmula de creación de información que reúne en un espacio vital a todos los protagonistas de los medios digitales, como precisa Ruiz de Elvira:

\begin{abstract}
Eso es Internet para los medios de comunicación: información digitalizada, interactividad entre los productores (periodistas) y los consumidores (lectores), instantaneidad, globalidad, valor añadido y algo, si cabe, aún más atractivo: constituir un soporte multimedia en el que se pueden integrar textos, fotos, infografía animada, sonido y vídeo. Es decir, la fusión en un solo medio del papel, la radio, la televisión, el cine, la fotografía e, incluso, el teléfono (Ruiz de Elvira, 1997).
\end{abstract}

El concepto de actualidad cambia en la información y consecuentemente en la documentación. De una edición, o dos, se pasa a otra digital actualizada constantemente. Es la información personalizada, servida en el correo del lector o en avisos que recibe cada cierto tiempo. Y en ese proceso ¿cómo actúa la documentación?

Hasta la llegada de Internet, el sistema de trabajo documental apenas avanzó. Su tarea principal sigue siendo el recorte de información de los principales medios, su clasificación y su custodia en sobres que ordenan alfabéticamente. El sistema deja de ser útil cuando la velocidad de la información es mayor. De una se pasa a varias ediciones y luego a una actualización constante. Está claro que poner los textos en sobres ya no sirve. Es tiempo de pasar a la acción. Así, los documentalistas 
colocan los contenidos en bases de datos, que el periodista puede consultar en pocos segundos. Además, hace un seguimiento a la información y prepara informes que luego puede consultar el periodista. Y, lo más importante, se convierte en parte de la estructura de la información. ¿De qué manera participa el documentalista en este nuevo sistema de información? Aportando elementos complementarios que apoyan, sustentan y dan vida a lo que el periodista digital ofrece. Un ejemplo clarificador puede hacernos entender qué valor aporta el documentalista en este nuevo tipo de información. Cuando se produce un atentado terrorista, el periodista digital cuenta en su información aquellos hechos noticiosos que reflejan la realidad del evento que está presenciando y/o cuenta con las opiniones de testigos presenciales. Como es una información que va generando otros elementos informativos, el periodista sigue la narración e introduce en el periódico digital nuevos datos que se producen conforme va avanzando el proceso noticioso. Mientras, desde el centro de documentación, que previamente ha elaborado y actualizado un informe de atentados terroristas, el documentalista aporta al periódico digital los datos que el periodista no recuerda y que completan un ciclo informativo global. En este sentido, el periodista se ocupa y preocupa de la información puntual que está sucediendo y el documentalista aporta los elementos retrospectivos que alimentan al lector y le sitúan dentro de un contexto informativo.

Llegados aquí, es preciso recordar un aspecto significativo. Antes de la llegada de los periódicos digitales, esa información se había realizado de otra forma: el periodista recoge la información en el lugar de los hechos, regresa al periódico, pide los últimos atentados al centro de documentación y elabora con todo ello una única información. Ahora no. El periodista digital envía la información de manera directa a su periódico y no tiene contacto con el documentalista que está completando su información. El proceso se acorta y permite mejorar y actualizar la información, ya que ambos hacen un seguimiento próximo a lo que está sucediendo.

El documentalista se ha preocupado durante muchos años de verificar la información y proporcionar los antecedentes de la información. En un informe que se considera la primera síntesis de los estudios de documentación periodística (Desmond, 1934) se recogen, además, otras características que han sustentado la documentación durante siglos hasta la llegada de Internet:

1. Mejorar la presencia de información local.

2. Adelantar la preparación de materiales y sugerir contenidos.

3. Dar información a los editorialistas.

4. Facilitar el material especializado.

5. Conservar la documentación valiosa.

6. Dar buena imagen del periódico.

7. Servir al departamento de publicidad.

8. Servir al departamento de difusión. 
La mayoría de estas funciones se siguieron realizando hasta finales del siglo pasado, pero la tecnología y la presencia de los medios en Internet dio otras prioridades al documentalista, que mira más hacia el futuro y apenas le queda tiempo para analizar de forma retrospectiva la información que se genera cada día y que hasta entonces tenía tanta importancia. Ahora se requiere otro planteamiento documental.

¿Qué gana el lector con este nuevo proceso? Más velocidad en la recepción de la información y sobre todo mayores contenidos. Y lo que es más importante: una actualización que el periódico digital ofrece a sus lectores, que pueden estar conectados o recibirla a través del correo, o de algún tipo de alerta. El proyecto final de información es, por tanto, totalmente diferente. Hasta la llegada de los periódicos digitales, los lectores tenían en la televisión y en la radio su manera más cercana y directa de estar informados. En la actualidad, aquéllos que trabajan de manera directa conectados a Internet pueden estar igual, o más informados, que los que escuchan la radio. Y todo gracias a la combinación de dos elementos: la información puntual del periodista que está en el lugar de los hechos con su ordenador portátil y el documentalista que hace un seguimiento puntual de lo que sucede y aporta los elementos informativos complementarios. El resultado final está claro: más cantidad, mejor servida e información más actualizada. Dicho en palabras de un experto, Gumersindo Lafuente, entonces director de elmundo.es: El control que nuestros lectores ejercen sobre el rigor y la calidad de la información es mucho más directo que el resto de los medios tradicionales. En el caso de los grandes medios, trabajar de forma estrecha con los redactores que salen al lugar de los hechos a fin de obtener la parte central de los hechos. El resto lo pondrá el documentalista.

\section{ALTERNATIVAS A LA GESTIÓN DOCUMENTAL EN LOS MEDIOS DE COMU- NICACIÓN: EL LECTOR, PROTAGONISTA}

Los dos elementos significativos de actuación creativa en el proceso informativo y documental de la información actual han abierto nuevos frentes al periodismo. Por esa puerta se han colado dos tipos que quieren y forman parte ya de los contenidos: el experto y, especialmente, el lector. El primero de ellos, es una extensión del tradicional periodista o colaborador que aportaba mediante artículos de opinión y reflexión, alternativas a la información que cada día creaba el periodista. Esta figura apenas cambió con la llegada de Internet. Si acaso, hubo de mejorar su lenguaje, adaptarlo a lo que los receptores pedían y hacer los textos más sencillos y digeribles para seguir contando con el favor de los lectores. Más de lo mismo, pero en diferente envase. Y, quizás, resultados similares, ya que los lectores de medios digitales prefieren la información de actualidad que los artículos de opinión de expertos en la materia.

El otro frente, el del lector, es el que ha revolucionado de forma considerable la información. Varias son las etapas que ha protagonizado:

1. Lector receptor: comienzo de los periódicos digitales. Se conforma con lo que lee. 
Apenas usa los enlaces del hipertexto y se acerca al periódico a través de una o dos secciones, pero no lo toma como un todo.

2. Lector pensante: nace con el asentamiento de los grandes periódicos digitales y cree que la información tienen un valor, que le puede ayudar a crear su propia opinión y lee de forma secuencial el medio, parándose de forma especial en aquellas secciones o artículos que le resultan de más interés. El editor puede saber por el tiempo de permanencia en una sección qué tipo de información le interesa más a sus lectores.

3. Lector crítico: aparece cuando algunos medios han ido desapareciendo por su pérdida de credibilidad o porque no han sabido ofrecer la información tal y como la desean los usuarios. Se caracteriza por ser crítico con el medio que ve con asiduidad y rompe la barrera de un solo medio. Apuesta por ir a otros periódicos cuando el que consulta habitualmente no le satisface. Se trata de un lector que opta por varias publicaciones y que es difícil de encasillar.

4. Lector actuante: se ha ido configurando a lo largo de más de una década, en la que ha visto nacer, crecer y evolucionar los periódicos digitales. Ha estado ausente, mirando desde la distancia y, por fin, ha entendido que puede ser parte de la información. En los primeros momentos, participando a través de correos electrónicos que se empleaban en la elaboración de la información, una vez contrastados los datos por el documentalista y, luego, entrando en directo para preguntar a algún personaje famoso.

5. Lector integral: forma parte del sistema actual. Se ha convertido en una especie de colaborador que envía información con el fin de que se tome como referencia para elaborar el producto final o que, al menos, se incluya una parte o la totalidad de la misma.

Este último ha sido el que se ha establecido como lector principal en la mayoría de las publicaciones digitales. Lee los periódicos en Internet y al mismo tiempo siente que es partícipe y protagonista de la información.

¿Cómo se llega a esta situación? Es preciso rastrear en la historia reciente para ver cómo el lector se convierte en el protagonista principal de la información digital. Cuando a finales de los noventa los periódicos digitales estaban estableciendo sus reglas de actuación, el profesor Marcos Recio consideró un aspecto importante presentar la información bajo el Triángulo de Información Electrónica, que ya incluía como aspecto fundamental al lector. Entonces, ya era consciente de que si no se aplicaban métodos para dar protagonismo al lector, éste no se acercaría a una pantalla a leer la información. Surge entonces el Diario Yo, que Negroponte propone en su libro Ser Digital. Más tarde se empieza a hablar del periódico personalizado, creado en función de los gustos y las necesidades de sus lectores y el último escalón eleva a los altares al lector. En este sentido, estos medios incluyen una gestión de la do- 
cumentación, pues se trata de elaborar un periódico para un grupo de usuarios que son muy exigentes. Como ha afirmado Marcos Recio (1999: 84-85), en esa convivencia entre periódicos digitales, hechos por y para una persona y los de información general, con su propio centro de documentación trabajando para ofrecer la mejor información, el lector determinará cuándo y por qué elige uno u otro.

Los elementos diferenciales de la información se convierten en el punto de calidad de los periódicos digitales. Lo que aportan los documentalistas son sólo una pequeña parte de lo que al final se considera la información global; es decir, el producto informativo que se ofrece al lector como contenido de actualidad, aspectos informativos de calidad, medio de creación de opinión y recursos documentales para la formación del lector.

\section{TAREAS DOCUMENTALES PARA GANAR YIO PERDER LECTORES}

El lector es el protagonista porque los medios le han convertido en el centro de atención. Esta tarea es habitual en la documentación. Los servicios que gestionan las bibliotecas y centros de documentación tienen un fin claro: servir las informaciones que desean los usuarios y así lo han hecho con los periodistas que demandaban una parte de los contenidos a publicar. Los periodistas saben de forma certera que sin lectores, no son nadie. En este océano de datos que son las publicaciones digitales, el Documentalista de Información Electrónica (DIE) selecciona, ordena y clasifica los contenidos para hacer más ¿fácil? el trabajo del periodista.

Entonces, ¿en qué situación se encuentran los periódicos digitales respecto a sus lectores? ¿Cómo afrontan la información para tenerlos como lectores fijos? ¿Qué relación tienen con otros medios? ¿Quién decide sobre los contenidos finales? No hay una respuesta milagrosa porque esta gran ola de medios apenas ha llegado a la orilla cuando otra ola más grande y poderosa se acerca.

La manera de entender algunas posibles respuestas pasa por situar el punto de equilibrio de los periódicos digitales frente a otros medios, sobre todo en los tres últimos años, donde las convulsiones han sido muy fuertes. En Internet, los periódicos son un elemento más de valor frente a otros que ofrecen más y mejor contenido. Tómese como ejemplo los siguientes casos: Myspace, Wikipedia, YouTube, Daily Motion, Flickr, Netvibes o Facebook. Todos estos sitios tienen algo en común: que los servicios que ofrecen tienen como misión satisfacer al usuario; de esta forma se convierte en el centro de atención de estos lugares de Internet.

Estos lugares suponen un auténtico peligro para los periódicos digitales. Se trata de tiempo. Si los lectores acuden a otro sitio donde, además de información, se incluyen servicios, terminarán por olvidarse de los periódicos digitales y acudirán a comunidades virtuales o a centros donde la información es sólo una parte importante y no la esencial como ha sido hasta ahora el modelo de negocio que ofrecían los periódicos en Internet. 
La ventaja es perceptible. Antes, los medios reaccionaban tarde y, cuando lo hacían, apenas tenían movilidad para enfrentarse o complementar a otros medios. En la actualidad se han convertido en modelos que fluctúan en función del mercado. Si los lectores acuden a ver o descargar vídeos de YouTube, el periódico puede hacer lo mismo con su información. Al redactor se le dota de una cámara y una parte de la información se coloca como imagen para descargar.

Este proceso ha pasado por varias etapas. Una de las destacables, por su calado social, fue la creación de un periódico de, por y para los lectores, Ohmynews. La intención era ofrecer un reportero universal, según su redactor jefe, Yu-jin Chang, que entonces apostaba por un periodismo joven, igualitario, y muy informal y desenfadado (La Vanguardia, 2006). El requisito clásico de escuchar al lector y darle protagonismo se hizo realidad en este medio, ya que según este periodista los lectores no se conforman con que les llegue la información, pues prefieren dar su propia versión de los acontecimientos. Esta situación rompe con las fórmulas tradicionales del periodismo, pues los receptores se convierten en los protagonistas de las informaciones que les son próximas y que les resultan más fáciles de escribir, tales como los acontecimientos de la vida cotidiana, los escándalos o el tiempo, que serían las secciones que más informaciones reciben. Pero el periodismo serio es algo más que todo eso. ¿Y la opinión? ¿Y la información política? ¿Y los resultados económicos y los informes y balances de la situación financiera de una empresa o país? Aún es pronto, pero ya hay quien asegura que esto no es periodismo y que pronto desaparecerá.

La verdadera preocupación para los periódicos son los sitios de Internet, creados hace poco y con un verdadero poder de convocatoria. Para evitar la pérdida de visitas, que se convierte en menos lectores, algunos periódicos digitales están utilizando las mismas herramientas que estos lugares de Internet. Si no puedes vencer a tu enemigo, únete a él o utiliza sus mismas tácticas. Eso debió pensar el editor del periódico The Sun cuando lanzó la propuesta de MySun, un espacio dentro del periódico en el que se invita a los lectores a que sean los nuevos editores del medio digital y envíen sus artículos, comentarios, fotografías, etc. Así, mientras lugares como MySpace o YouTube quieren compartir vídeos con sus usuarios y para ello reciben miles de vídeos de diversos contenidos elaborados por los propios usuarios, el periódico británico más leído optó por la misma fórmula para captar lectores: Queremos tus vídeos y tus mejores historias (Tubella, 2006). Y todo ello, gratis para los medios, que si bien no están dispuestos a pagar, sí, al menos, reconocen los derechos de autor sobre el material que envían los lectores al periódico.

En medio de estas dos propuestas caben otras muchas alternativas y ensayos por parte de los editores de periódicos digitales. Para la documentación son años difíciles, pues ha de variar su rumbo, ya que lo que demandan los lectores son informaciones de cada día, no artículos de expertos, teorías, ciencia, opinión, reflexión. Ése es el periodismo de antes; el que viene es más participativo y es de todos los lectores. Ellos tienen la última palabra. 


\section{CONCLUSIONES}

Fuera tópicos sobre lo que puede pasarle al periodismo digital. Ya hace tiempo que algunos teóricos han enterrado, de manera acertada, la muerte y el resurgimiento del periodismo impreso frente al digital. Lo que se propone en este artículo es una nueva vía hacia un periodismo de servicios que tendrá diferentes maneras de expresarse, con un punto común: una pantalla. ¿Será la del ordenador? Quizás la del teléfono móvil o la de la televisión. No hace falta esperar mucho. En breve, tendremos una respuesta.

Mientras tanto, algunas grandes editoriales mueven sus fichas. El ejemplo más reciente es el de The New York Times, que contrario a lo que se explica en las líneas precedentes, ha decidido vender sus cadenas de televisión para apostar por el periodismo digital en Internet. ¿Es una retirada a los cuarteles de invierno? ¿Le seguirán otras empresas? ¿Habrá más descentralización de medios en grandes grupos?

Otros medios apuestan por lo contrario. Es decir, emplear técnicas similares a las de otros sitios de Internet que funcionan. El caso ya citado de The Sun y otros medios del grupo del empresario australiano Rupert Murdoch son un ejemplo, incluido MySpace, que compró el magnate de los medios de comunicación como recoge Tubella:

Las audiencias de hoy se revelan cada día más participativas. Baste como botón de muestra que, a raíz de los atentados del año pasado en la capital británica, la BBC recibió más de 20.000 e-mails. Un fenómeno en alza que el magnate de la comunicación más poderoso del planeta, Rupert Murdoch, ya avistó al adquirir MySpace (Tubella, 2006).

Es tiempo para estar despierto, de mirar lo que otros hacen en su apuesta por extender su imperio mediático. Los periódicos han de continuar su labor de información, crear opinión y ofertar servicios a través de la publicidad para continuar generando recursos financieros que hagan viable la publicación. Mientras tanto, cederán una pequeña parte de su parcela a los lectores, a los que han de incluir como elementos informativos que autogeneren parte de sus contenidos. El problema será saber dónde colocar la barrera entre los contenidos profesionales y los que demandan los lectores.

Y, por lo que respecta a la documentación, debemos indicar que siempre ha ido de la mano de la información, pero ahora tiene un camino diferente: mientras los periodistas ofrecen cada vez más información de actualidad, los documentalistas han de rastrear Internet para facilitar esa búsqueda a los redactores. Y, al mismo tiempo, asentar los contenidos que apenas tienen valor de cara a un futuro incierto en el que la información apenas sirve un par de horas. En un estado de constante movimiento, periodista y documentalista seguirán ejerciendo labores informativas que otros medios digitales no pueden ofrecer. Ése es el verdadero periodismo y el que tiene las puertas abiertas para los lectores de siempre. Esta reflexión del profesor Luján Za- 
vala es un excelente colofón a lo que la documentación es al periodismo y viceversa: Los periodistas en Internet no estamos con los brazos cruzados esperando la información de la redacción. En la medida de nuestras posibilidades, seguiremos investigando, analizando, verificando, ponderando, contrastando y publicando sin perder la visión integral de la sociedad y el hombre.

\section{REFERENCIAS BIBLIOGRÁFICAS}

BERNERS-LEE, T.; HENDLER, J.; LASSILA, O. "The Semantic Web". Scientific American (May 2001), Disponible en: http://www.scientificamerican.com.

CODINA; L.; ROVIRA, C. La Web semántica. Universidad Pompeu Fabra, Departamento de Periodismo y Comunicación Audiovisual. Disponible en: http://www.semanticaweb.net

DESMOND, R. W. "Newspaper reference Metohds". The American Journal of Sociology (Noviembre 1934), Vol. 40, $\mathrm{n}^{\circ} 3$.

GALDÓN LÓPEZ, G. Perfil histórico de la documentación en la prensa de información general: 1845-1984. Pamplona: Eunsa, 1994.

- (1986) El servicio de documentación de prensa: funciones y métodos. Barcelona: Mitre.

LUJÁN ZAVALA, J. C. "Periodismo digital y la relación con los usuarios en la era digital”. Razón y Palabra. Disponible en http://www.razonypalabra.org.mx/anteriores/n30/jlujan.html

MARCOS RECIO, J. C. La documentación electrónica en los medios de comunicación. Madrid: Fragua, 1999.

NATHAN, G. J. "Journalistic Morgues". The Bookman (Agosto 1910), Vol. 31, pp. 597-599.

NEGROPONTE. N. Ser Digital. Editorial Atlántida, 1995.

RUIZ DE ELVIRA, M. En pugna con los diarios. En El País, 18 de diciembre de 1997.

TUBELLA, P. El periodismo participativo gana adeptos en Inglaterra. Disponible en: http://www.lavanguardia.es. 


\section{Breve semblanza de los autores}

Juan Carlos Marcos Recio es Profesor Contratado Doctor del Departamento de Biblioteconomía y Documentación de la Facultad de Ciencias de la Información de la Universidad Complutense y Master in Library \& Information Studies por el University College of Wales y por la Universidad Antonio de Nebrija de Madrid. Anteriormente ha sido profesor de la Universidad de Valladolid, la Institución Universitaria Mississippi y la Universidad Antonio de Nebrija. Es autor de tres libros individuales y de numerosos artículos en revistas españolas y extranjeras relacionados con su especialidad. Ha trabajado como periodista y documentalista en periódicos y revistas españoles y mantiene otras líneas de investigación como la publicidad, las relaciones públicas y la educación a distancia.

Concepción Edo Bolós es Profesora Titular de Periodismo en la Universidad Complutense y, anteriormente, profesora de la Universidad Carlos III de Madrid. Su investigación se centra en el estudio de la prensa diaria y los cibermedios. Ha participado y participa en proyectos de investigación de financiación pública y privada, ha publicado tres libros como autora individual y ha participado en dieciocho obras colectivas. Ha escrito decenas de artículos en revistas académicas y ha participado como ponente en congresos nacionales e internacionales. Es subdirectora de la revista académica Textual\&Visual Media, miembro del Consejo de Redacción de la revista Estudios sobre el mensaje periodístico de la Universidad Complutense, miembro del Consejo Editorial de la web "Sala de Prensa" y secretaria de la Junta Directiva de la Sociedad Española de Periodística.

Jesús Flores Vivar es Profesor de la Universidad Complutense de Madrid, de la Universidad Antonio de Nebrija y de la Universitat Oberta de Catalunya. Profesor y conferenciante invitado en otros centros e instituciones nacionales e internacionales, es coordinador del Observatorio de Periodismo en Internet y del proyecto Cybermedia (Plan Nacional I+D) y participa en Proyectos de Innovación y Mejora Docente. Es autor y coautor de libros y artículos especializados en Ciberperiodismo y ha organizado y dirigido siete congresos internacionales sobre Periodismo e Internet. Ha trabajado en prensa, radio y medios digitales. Forma parte del Comité Científico de Tesis OCS y colabora en la web del Poynter Institute. Aborda las siguientes líneas de investigación: modelos de negocio en el Ciberperiodismo; blogs y Web 2.0, gestión del conocimiento y formación e-Learning.

Pedro García-Alonso es Profesor Contratado Doctor de Empresa Informativa en la Universidad Complutense de Madrid, M.B.A. por el Instituto de Empresa y doctor en Periodismo y Filosofía. Ha sido profesor en distintas universidades de Periodismo, Marketing, Comunicación Audiovisual, Ética Empresarial y áreas afines. Es miembro Fundador de 'European Business Ethics Network' en España y Secretario de la Asociación de Periodistas Bloggers. Autor de libros manuales universitarios como "Empresa Informativa. Los medios en 2007", "Tesis y Doctorado. Espacio Europeo de Educación Superior", "Teoría y Práctica de la Dirección en la Empresa Informativa" y "Organización y Gestión de la Empresa Informativa", ha participado en más de veinte congresos en España, México, Chequia y Portugal. Participa en el Programa de Evaluación de la Calidad Docente del Vicerrectorado Complutense.

David Parra es Doctor en Ciencias de la Información por la Facultad de Ciencias de la Información de la Universidad Complutense y Diplomado en Derecho por la UNED. Es autor de cinco libros sobre tecnologías de la información aplicadas al ámbito del periodismo. Asimismo ha participado en alrededor de quince obras colectivas, ha escrito decenas de artículos en re- 
Juan C. Marcos, Concha Edo, Jesús Flores, Pedro García-Alonso, David Parra

vistas científicas y ha participado en distintos congresos nacionales e internacionales. Sus líneas de investigación se vienen centrando durante los últimos veinte años en la convergencia multimedia, examinando fenómenos como el videotex, Internet, la telefonía celular, Internet Móvil y el ciberperiodismo.

(Recibido el 7-12-07, aceptado el 21-03-08) 